\title{
REFLEXIONES
}

\section{Explorando el Psicoanálisis Multifamiliar Reflexiones en torno a la forma de entender el sufrimiento de los pacientes}

\author{
Arturo Marín Arévalo \\ IPR, Madrid
}

\section{La formación de un psicoterapeuta.}

Sin duda la formación de un psicoterapeuta nunca debe considerarse como finalizada, de hecho, puede llegar a estar en continuo cambio y actualización. Pero creo que los primeros años de experiencia son los que determinan con más fuerza nuestra identidad profesional, más allá de los procesos de transformación que podamos tener a lo largo de nuestra carrera. En mi caso, se podría decir que mi camino acaba de comenzar. Durante 4 años como psicólogo residente he podido entrar en contacto con una gran variedad de pacientes, contextos terapéuticos, supervisores y equipos de trabajo. Paralelamente, he intentado ampliar mis conocimientos teóricos profundizado principalmente en el modelo relacional del psicoanálisis, con el cual me siento plenamente identificado.

Habitualmente ha sido complicado mantener la coherencia entre aquello que voy aprendiendo con mis lecturas de Winnicott, Kohut o de la teoría intersubjetiva, por poner ejemplos, con aquello que he ido experimentando día a día en mi práctica clínica. Habitualmente la disonancia ha sido intensa. Me ha resultado difícil por ejemplo tomar conciencia de las repercusiones que tiene para el paciente el trato que reciben en el ámbito hospitalario. El paciente muchas veces quedaba totalmente alienado por el diagnóstico, la farmacología y el ser percibido como un "enfermo mental". En estos casos la expresión del sufrimiento a través de los síntomas simplemente era considerada como una expresión de "locura" producto de un fallo en la química cerebral. También me he enfrentado a las limitaciones para poder tratar con garantías a pacientes graves a nivel ambulatorio. He sentido que no se podía ayudar lo suficiente a personas con un intenso sufrimiento por la limitación de recursos. A pesar de ello, mi formación en el modelo relacional ha funcionado como una especie de base segura desde la cual poder soportar mejor mis frustraciones y a la vez autoafirmarme en mi manera de pensar. Recuerdo el alivio que sentí cuando leí el artículo de Coderch (2012) sobre la eficacia del tratamiento basado en los sistemas dinámicos no lineales, donde se argumenta la relativa importancia de la alta frecuencia de las sesiones para el cambio del paciente. 
Pero está claro que no todo ha sido negativo. En este periodo he podido aprender una gran diversidad de formas de trabajo. Creo que muchas de ellas han reforzado mi convicción en la perspectiva relacional. Por ejemplo, he tenido la suerte de entrar en contacto con el modelo sistémico a través del trabajo en infantil y en el Hospital de día, lo cual me ha permitido ver la importancia del trabajo directo con familias. El formato familiar nos permite intervenir directamente con la matriz relacional del paciente, más allá del campo intersubjetivo creado únicamente por el paciente y terapeuta. Conforme han pasado los años, también he podido tomar conciencia de la importancia del trabajo grupal. Es difícil tener como base teórica conceptos como intersubjetividad y no tener en cuenta el poder del grupo como espacio de apertura y encuentro entre subjetividades.

Creo que todo este bagaje de experiencia clínica y aprendizaje teórico ha sido sorprendentemente integrado en una de mis últimas rotaciones como psicólogo residente. Justo al final de este periodo he tenido la oportunidad de viajar tres meses a Argentina para entrar en contacto con el psicoanálisis multifamiliar. Se me presentó la oportunidad de participar de una comunidad terapéutica donde la forma de trabajar con el paciente estaba en completa sintonía con mi identidad como psicoterapeuta, algo que no había ocurrido en mis 4 años de residencia. Era la oportunidad de poder integrar mi formación en psicoanálisis con el trabajo directo con familias y además poder hacerlo en un contexto de terapia grupal. Pero la experiencia fue mucho más allá. Principalmente ha sido un periodo de reflexión profunda sobre la forma de entender la "locura" y la influencia determinante que tiene nuestra "mirada" sobre el paciente a la hora de facilitar o impedir su desarrollo como persona.

\section{El Dr. Badaracco y el Psicoanálisis Multifamiliar.}

Resulta difícil resumir en unas pocas líneas en que consiste el psicoanálisis multifamiliar, iniciado en los años 50 por el doctor Jorge Luis García Badaracco. Cuando entré en contacto con su teoría y su trabajo no lo sentí como algo ajeno, sin duda estaban íntimamente asociados con la perspectiva relacional del psicoanálisis y con el modelo sistémico. Por ello pude integrar a la perfección su teoría y enriquecerla con mis propios conocimientos.

Los inicios del psicoanálisis multifamiliar se encuentran en el ámbito psiquiátrico y hospitalario donde predominaba la visión del paciente como un enfermo crónico e incurable. En este contexto, el doctor Badaracco, en ese momento psiquiatra del Hospital Borda en Buenos Aires, intenta crear un espacio donde poder ofrecer un ambiente más humano a los pacientes. Comenzó a reunirse con ellos de forma espontánea en un espacio común y compartido por todos, incluidos los profesionales. Su objetivo era transformar hospital psiquiátrico en una comunidad terapéutica donde poder intervenir con el paciente desde una mayor cercanía y horizontalidad. Pronto, aquellos pacientes graves, considerados "locos" y desconectados del medio, empezaron a hacer intentos por comunicarse con él. Los comportamientos y expresiones que eran consideradas por otros profesionales como 
síntomas de locura, eran recogidos por Badaracco como intentos de comunicación. Esta actitud permitía buscar el sentido y el significado de estas manifestaciones (García Badaracco, 2006a).

Pero los comportamientos de los pacientes habitualmente implicaban actitudes agresivas y retadoras que parecían incomprensibles en un primer momento. Otras veces los profesionales se veían atrapados por el paciente en paradojas enloquecedoras que les impedían pensar e intervenir de manera coherente. Badaracco empezó a interpretar estos comportamientos como la transferencia psicótica en el grupo de las experiencias familiares que habían hecho enloquecer al propio paciente. Sin duda sus síntomas eran formas de expresar experiencias traumáticas que no habían podido metabolizarse y que solo podían actuarse: lo que había sido sufrido de manera pasiva en sus familias era repetido de manera activa con los compañeros y profesionales de la comunidad terapéutica. Ante este descubrimiento se planteó la importancia de incluir a la familia en los encuentros. El objetivo era intervenir de manera más directa en este tipo de interacciones. Gracias a ello se empezó a observar que los familiares también cargaban con un intenso sufrimiento. Expresaban defensas rígidas que inconscientemente hacían enloquecer al paciente, provocando a su vez que el paciente contraatacase y se convirtiese en alguien enloquecedor para la propia familia. Se observó por tanto la causalidad circular del problema y las interdependencias reciprocas dentro del sistema familia. En ese punto es donde nace el psicoanálisis multifamiliar como tratamiento de pacientes graves a nivel grupal y familiar.

Badaracco (2006f) parte de la premisa de que la mente humana se desarrolla dentro de un campo de interdependencias reciprocas, donde los comportamientos de los individuos estarán mutuamente condicionados y determinados. Considera que la base de la patología del paciente grave está la simbiosis patológica que establece con la familia (García Badaracco, 1989). Si bien la relación simbiótica es un punto de partida necesario para facilitar el desarrollo normal, la simbiosis patológica implica una dependencia rígida que impide el desarrollo de los recursos necesarios para alcanzar la autonomía. En este caso la simbiosis se establece con un "objeto enloquecedor" que basa su relación con el sujeto en la satisfacción de sus propias necesidades, tratándole más como una posesión que como un sujeto diferenciado. Esto provoca que se convierta en una presencia invasora, exigente y rechazante, que solo se muestra disponible si hay una adaptación a sus deseos y una renuncia por parte del sujeto a la expresión de su verdadero self. Por ello la única defensa posible ante esta "presencia enloquecedora" es la identificación total con ella y la distorsión del self. Como consecuencia de ello, las relaciones que se establecen con los otros se basan en la actuación sin reflexión, lo que da lugar a la repetición compulsiva de patrones, a la complementariedad rígida entre sujetos y a los roles intercambiable de víctima-agresor. Badaracco (2006e) utiliza la metáfora de "los otros en nosotros" para describir estas presencias internas que persiguen sin descanso y exigen un permanente sometimiento. El sujeto queda "habitado" por presencias atemorizantes que congelan su desarrollo y le condena a una dependencia irresoluble. 


\section{La psicopatología grave y "la mirada del otro".}

Si nos basamos en la teoría intersubjetiva se podría decir que Badaracco describe contextos intersubjetivos donde el self del sujeto se encuentra constantemente al borde de la aniquilación (Orange, Atwood \& Stolorow, 2012). La única vía posible de supervivencia es la sobreadaptación a los deseos de las figuras de apego, quedando inevitablemente alienado por el ambiente familiar. Para llevar a cabo este proceso es crucial la disociación. Esta defensa permite organizar las relaciones enloquecedoras de las que uno no puede escapar. Esto puede observarse cuando se analiza el apego desorganizado. En este tipo de apego, el cuidador es percibido como fuente de terror, pero a la vez es experimentado como la única figura que puede proporcionar seguridad y vitalidad. Esta paradoja provoca en el infante el despliegue de conductas confusas, incoherentes y contradictorias. Por ello es necesaria la disociación como la única forma de evitar el caos y la aniquilación del self. El fallo en la disociación es lo que provoca los síntomas de "locura" en forma de delirios, alucinaciones o acting out. Estos síntomas pueden ser entendidos como defensas extremas para mantener con vida al self cuando los intentos de organización fracasan y se vuelve a re-experimentar el caos provocado por el poder enloquecedor del otro.

Desde una perspectiva sistémica, se podría decir que la disociación y sobre-adaptación del sujeto se plantea como el resultado de una exigencia familiar destinada a mantener un equilibrio patológico. Se plantean conceptos como pseudo-mutualidad o mistificación para explicar cómo el sujeto se adapta a la patología familiar. Esta adaptación supone que el sujeto tiene que soportar sobre sus hombros toda la "locura" familiar, lo que implica un estado de tensión y de negación de sí mismo que sin duda pone en peligro la integridad de su self. Por otro lado, vemos como la Escuela de Milán considera al paciente designado como el perdedor del juego familiar, siendo sus síntomas psicóticos una forma de protesta ante la situación (Selvini Palazzoli et al, 1990). Los síntomas se acaban convirtiendo en una estrategia más dentro del juego. Por tanto, la amenaza de aniquilación del self no solo proviene del posible caos en la relación, también proviene de la sobre-adaptación absoluta al otro. Teniendo en cuenta todo esto, creo que los síntomas psicóticos pueden plantearse como la rebelión encubierta que el sujeto realiza en contra de las reglas impuestas por la familia. Estos síntomas se presentarían como la única forma posible de expresar y representar un sufrimiento insostenible en un contexto que niega sus necesidades, sus percepciones y le anula como sujeto diferenciado.

Ya Freud planteó la psicosis como la rebelión del Ello ante la realidad. Pero no llegó a desarrollar lo tremendamente alienante que puede llegar a ser la realidad transmitida por la familia. Se podría decir que la rebelión la realiza el verdadero self en un acto de "fortaleza", luchando en contra de una realidad invalidante que le conduce hacia la disolución y la aniquilación. El sujeto renuncia a intentar una sobre-adaptación imposible, prefiriendo 
sobrevivir en el caos relacional con ayuda de los síntomas. Por ello en la psicosis encontraremos una expresión distorsionada del self en forma de omnipotencia, así como fenómenos de control o influencia como expresión del control familiar, o sensaciones proyectadas al exterior de confusión y persecución como expresión del temor constante a una inminente aniquilación.

Esta "rebelión" hace que los síntomas, como el delirio o la alucinación, supongan una amenaza para la familia, ya que implican la "denuncia" de un sufrimiento compartido por todos, sufrimiento que debe ser ocultado y negado para la supervivencia y estabilidad del sistema. Por ello esta "denuncia" solo puede expresarse de manera distorsionada, con síntomas incoherentes e incomprensibles para los otros. El objetivo de esta incomprensibilidad es evitar el peligro de destrucción que supondría para el sistema destapar de manera clara y abierta toda "locura" familiar oculta. Basándonos en todo esto, creo que el síntoma psicótico funciona como una solución de compromiso entre la expresión de protesta de un self al borde de la aniquilación, y el sometimiento a las reglas implícitas de una familia de la que no se puede prescindir. Pero el componente de protesta y denuncia puede ser fácilmente silenciado por el sistema. La incomprensibilidad de las expresiones permite etiquetar de "loco" al sujeto, lo que sirve para restaurar el equilibrio inicial y la máscara de normalidad familiar, dejando al sujeto nuevamente invalidado en su expresión. Esta es la lógica descrita en el doble vínculo (Bateson, 1971) donde el sujeto queda desorganizado y bloqueado en una paradoja irresoluble que no puede ser denunciada abiertamente y de la que no se puede escapar bajo ningún concepto. La psicosis se presentaría como la solución para mantener a toda costa una relación imposible, y así evitar una situación aún peor para el sujeto: la ausencia de relación y el vació.

Este tipo de ambiente familiar está lejos de ser un contexto intersubjetivo de validación y reconocimiento mutuo donde poder elaborar y simbolizar la experiencia del self (Benjamín, 1997). En el caso de la psicosis el contexto intersubjetivo se basa en la alternativa entre el caos o la alienación. La única forma posible de comunicación de los estados del self es a través de la "locura". Los síntomas aparecen como formas de expresión concreta a modo de ecuación simbólica (Segal, 1989). Por ejemplo, un sujeto puede tener la necesidad defensiva de desarrollar un self omnipotente ante la sensación de inminente aniquilación psíquica en un contexto familiar determinado. Puede concretar esta necesidad en un delirio diciendo "soy dios", para luego comportarse como tal en dicho contexto. Por asociación, puede llegar a expresar su capacidad para leer la mente del otro, para conseguir que "el mundo cambie" a su antojo, o para controlar su entorno de manera mágica. En este caso no hay un espacio relacional de confianza donde poder co-construir sus sensaciones para que puedan ser metabolizadas e integradas. Un espacio potencial de este tipo permitiría experimentar estas mismas sensaciones de forma distinta. Se llegaría a un punto en el que se podría expresar: "en mi familia yo siento que debo poder con todo" o "tengo la necesidad de sentirme que puedo con todo", aceptando a la vez el duelo, las limitaciones y los aspectos dolorosos de la situación. En este caso, el sujeto realiza su expresión de manera simbólica, lo que permite 
comunicar y compartir con el otro su propia experiencia de una forma comprensible. Esta diferencia de contexto y de funcionamiento es la que deja al psicótico fuera de la realidad compartida a nivel social, siendo considerado simplemente como un "loco".

El problema aparece cuando el profesional también considera la psicosis como una locura incomprensible, participando involuntariamente de la alienación familiar. Cuando desde la psiquiatría se consideran los síntomas como expresiones carentes de sentido y significado, el intento distorsionado de comunicación del sujeto queda invalidado. En este caso, el sujeto necesita de un espacio intersubjetivo donde su expresión pueda ser considerada como una manifestación con un sentido determinado. Esa es la función que tienen los grupos multifamiliares y el encuentro terapéutico con pacientes psicóticos. Sin duda lo más importante con estos pacientes es tener en cuenta la disposición y la mirada de los otros ante la "locura", de ello dependerá la creación de un espacio terapéutica donde los síntomas sean considerados como algo más que la manifestación de un desequilibrio químico sin sentido, como ocurre en muchos ámbitos psiquiátricos y hospitalarios.

Esta misma disposición terapéutica también puede ser aplicada a los acting out y a los comportamientos perversos o destructivos. Cuando Badaracco se encontró con este tipo de comportamientos en los grupos, no los consideró una expresión de "maldad" incurable sino la única forma que el sujeto tenía de expresar su sufrimiento. Se podían entender como una autoafirmación defensiva ante las presencias enloquecedoras que habitaban su mente. Por tanto, los actos destructivos pueden llevar asociados un reclamo afectivo que debe ser escuchado si se quiere ayudar. Esta actitud es la única manera de crear un encuentro donde pueda haber un reconocimiento mutuo, más allá del enactment irresoluble y de la complementariedad sadomasoquista entre sujetos (Benjamín, 1996). Este argumento también es propuesto por Kohut (1971) cuando plantea que detrás de la omnipotencia del narcisista existen necesidades infantiles no satisfechas. La ausencia de un objeto del self disponible obliga a crear una defensa narcisista, que a la vez se convierte en un reclamo para obtener la validación infantil negada en su momento. Es el terapeuta el que tiene que percibir más allá de la defensa para encontrar y validar las necesidades del paciente.

Creo que es Winnicott el primero el plantear esta actitud cuando habla de la "tendencia antisocial" en el niño (Winnicott, 1956). Para él la tendencia antisocial es una respuesta normal a la privación traumática del amor materno. Considera que el niño puede robar como forma de reclamar un amor perdido, o puede manifestar comportamientos destructivos como una forma de provocar una respuesta en el entorno. Este tipo de reclamos se observa perfectamente en el paciente infantil cuando aparecen comportamientos sádicos hacia los padres como forma de mantener el control de una relación frágil y perturbadora. En estos casos la clave es el fallo en el amor materno y la búsqueda desesperada del niño para recuperarlo. Cuando Winnicott (1971) habla del "uso del objeto" y de su "supervivencia", está planteando un espacio de encuentro intersubjetivo donde las autoafirmaciones del sujeto puedan ser reconocidas sin la necesidad de traspasar los límites del otro, transformando un 
acto destructivo en una demanda legítima que puede ser tenida en cuenta. Volvemos de nuevo al valor de la mirada del terapeuta cuando se enfrenta a estos comportamientos. De la misma manera que podía llegar a entenderse los síntomas psicóticos como simple "locura", los actos perversos pueden ser entendidos como "maldad" o como agresión descontrolada. Pero esta forma de entenderlo crea mayor distancia en el terapeuta lo que repite la sensación de privación, retroalimentando la tendencia antisocial.

Teniendo en cuenta todo esto, creo el aspecto más importante en el tratamiento de casos graves es la "manera de mirar" al paciente. Badaracco (2006b) considera que mirar a un "enfermo" como enfermo es potencialmente enfermante. Esta actitud impide el crecimiento del sujeto, negando la parte sana y el potencial oculto que permanecen intactos a pesar de su psicopatología. Badaracco (2006a, 2006b, 2006e) plantea el concepto de "virtualidad sana" para referirse a este potencial oculto aún por desarrollar. Este concepto se refiere "a que siempre existe en la mente humana una capacidad potencial para desarmar el poder enfermante de las presencias enloquecedoras" (Garcia Badaracco, 2006e). Pero esta capacidad solo puede desarrollarse en un ambiente que pueda reflejar con su "mirada" la parte sana, más allá de la "locura" manifiesta. No se trata de una posición ingenua donde se considera que todos los pacientes pueden "curarse", obviando su gravedad. Hay que tener muy en cuenta las limitaciones de cada uno para no crear falsa expectativas que hundan aún más a la persona. Creo que hay muchos pacientes tremendamente dañados que nunca llegarán a ser lo que pudieron ser si hubiesen vivido en otro entorno. Pero creo que considerar la "virtualidad sana" en todo paciente es una actitud necesaria en la terapia, es la única forma de establecer una "zona de desarrollo próximo" para el sujeto. Solo ésta "mirada" permite que el paciente pueda llegar a mejorar y desarrollarse como persona, sea cual sea la meta final. Para mí esta actitud supone un reto a nivel contra-transferencial cuando nos sentimos impotentes ante la gravedad del paciente y las limitaciones de los recursos para poder ayudarle.

Los grupos multifamiliares se plantean como un espacio donde esta "mirada" pueda ser una realidad. Se trata de encuentros donde la persona puede expresar tanto los aspectos "locos" como los sanos en un ambiente de confianza y seguridad. En este espacio la locura podrá ser transformada en algo significativo y el potencial sano en una realidad. El fin es que sus experiencias puedan ser elaboradas a través de la experiencia afectiva con los otros, más allá de comprensión cognitiva que proporciona la interpretación. Por otro lado, el grupo se plantea como un contexto ampliado, donde los miembros de las familias pueden abrir su mente y realizar cambios en la dinámica rígida y absorbente que les mantiene atrapados. Los distintos puntos de vista y formas de sentir permiten una apertura y la elaboración de las transferencias que se van desplegando en el grupo. Todo esto es lo que pude experimentar en los tres meses que pude asistir en la comunidad terapéutica y en los grupos multifamiliares. 


\section{Mi experiencia en la comunidad terapéutica.}

A mi llegada a la comunidad terapéutica, pronto pude comprobar lo diferente que iban a ser los tres meses que tenía por delante en comparación a mis anteriores experiencias como residente de psicología clínica. El ambiente que se respiraba en un principio me desconcertó y me creó inseguridades. Era llamativa la cercanía y horizontalidad con la que se trataba a los pacientes en los espacios comunes, donde los residentes teníamos bastante protagonismo. Me resultaba difícil definir la relación con ellos y saber cuál era mi lugar en la comunidad. Sentía que no podía recurrir a la seguridad que nos proporciona habitualmente nuestro rol profesional de psicoterapeuta a la hora de relacionarnos con los pacientes. Esta dificultad la sentía, por ejemplo, en los talleres que se realizaban después de los grupos, donde los residentes solíamos participar como si fuésemos uno más. También solíamos tomar café con ellos todos los días, incluso había días que cocinábamos y cenábamos todos juntos. Pero lo más significativo era que constantemente manteníamos conversaciones cotidianas y normalizadas con ellos. En este ambiente a veces sentía que la diferencia entre profesionales, residentes y pacientes se difuminaba. Pero lo que en un inicio parecía una ruptura de límites, pronto empecé a sentirlo como un espacio donde el paciente podía sentir al profesional mucho más cercano y más conectado con su sufrimiento. Más allá de una relación terapeutapaciente, las relaciones se convertían en un encuentro auténtico entre personas, donde el sufrimiento y los aspectos sanos podían ser compartidos y validados. En este contexto el rol de "enfermo mental" quedaba diluido, dejando paso a la persona.

Sin duda el ambiente que emergía de esta actitud era un claro representante de "mutualidad", tal y como la plantea Aron (1996). Las interacciones estaban totalmente basadas en la empatía y la cercanía con los otros. Pero como bien plantea este autor, esta actitud no implica renunciar a la asimetría necesaria que debe haber entre profesional y paciente. En la comunidad los profesionales tenían claro cuál era su lugar y su responsabilidad a pesar del trato cercano. Por ejemplo, había constantes reflexiones acerca del tipo de cercanía y de reciprocidad más apropiada para cada paciente. El fin era ser empático y respetuoso con las necesidades y angustias de cada uno de ellos, evitando una cercanía indiferenciada que pudiese perturbarles. Recuerdo una chica de 30 años con una terrible sensación de vacío e inadecuación que se relacionaba habitualmente desde una seducción bastante explicita con los hombres, principalmente con los residentes varones del centro, entre los que me incluía. Esta actitud seductora e invasiva inevitablemente provocaba un distanciamiento en nosotros. La percibíamos como alguien perturbador con quien no podíamos mostrarnos afectivos por miedo a que la relación se sexualizase. Por desgracia, ella percibía claramente esta actitud de distancia, lo que confirmaba su sensación de inadecuación y rechazo. Conforme se iban trabajando en los grupos estos aspectos relacionales, emergió en ella una necesidad de relacionarse con nosotros de forma distinta. Creo que empezó a mostrar su deseo de sentirse unida a un grupo que por edad y experiencia podía llegar a entenderla y aceptarla más fácilmente. En este punto es donde la actitud de cercanía y mutualidad era necesaria para poder conectar más fácilmente con esta nueva 
forma de relacionarse, diferenciándola de su parte más seductora. Si esta nueva forma de acercarse a nosotros se hubiese interpretado como otra seducción más, la oportunidad se habría perdido y se habría confirmado de nuevo su sentimiento de rechazo.

Como se muestra en este caso, vemos que la cercanía con el paciente podía convertirse en una fuente constante de conflictos. Los terapeutas y residentes se exponían de lleno a las transferencias pasionales y psicóticas de los pacientes. Por ejemplo, podían aparecer celos y envidia entre ellos cuando buscaban un trato diferencial con los profesionales. Por otro lado era habitual la idealización de un de los terapeutas y una mayor agresividad con respecto a otro, lo que producía un intento de escisión en el equipo. La clave para soportar todos estos procesos transferenciales estaba en el ambiente de sostén y contención que se creaba en la comunidad. El funcionamiento coordinado del equipo permitía mantener esta contención, controlando las escisiones y las paradojas enloquecedoras que muchos pacientes provocaban. A pesar de los esfuerzos, esta tarea no era fácil y por desgracia no siempre se conseguía. Aun así se consideraba que los conflictos que se producían en los espacios comunes no suponían algo negativo. Todo lo contrario, podían ser vistos como oportunidades para seguir elaborando las "presencias enloquecedoras" de los pacientes más allá del espacio grupal propiamente dicho.

Sin duda esta capacidad para introducirse de lleno en la locura y poder intervenir desde dentro, era el aspecto más relevante del tratamiento con los pacientes. Lo que me quedó claro desde el principio fue lo importante que era en este contexto no tener miedo a la psicosis ni a los acting out de los pacientes. Sin duda esto era todo un desafío para mí. Era necesario estar cien por cien disponibles al sufrimiento del otro, y para ello uno debía estar conectado permanentemente con emociones muy perturbadoras. El trato con los pacientes era continuo y muchos de ellos se mostraban, no solo seductores como el caso anterior, sino también amenazantes o enloquecedores. En esta situación era inevitable sentir la necesidad de actuar comportamientos o actitudes de distanciamiento afectivo. A mí personalmente me resultaba difícil mantener la mutualidad y la cercanía necesarias en el día a día. Me resultó especialmente difícil con una paciente psicótica que me introdujo de lleno en su delirio de filiación y megalomanía. Me sentía observado y controlado por ella y en el fondo, aunque lo intentaba negar, también me sentía amenazado. No solo sufría una identificación proyectiva enloquecedora, sino que sabía que había sido agresiva en el pasado con otros profesionales. Mi temor tenía una base real y concreta. Aunque mi temor me decía que me alejase, conseguí sobreponerme e intenté acercarme más a ella con el fin de normalizar la relación. Creo que fue la clave para que su comportamiento conmigo fuese menos invasivo y controlador. Al final creo que me hice más real para ella, ya no tenía la necesidad de depositar en mí sus propias proyecciones para crear una relación conmigo.

A pesar de que desde el ámbito hospitalario y psiquiátrico se esté muy familiarizado con la "locura", es raro que el profesional muestre este tipo se disponibilidad emocional. Creo que muchas de las actitudes de distancia afectiva y de complementariedad rígida entre psiquiatra 
y "enfermo mental", se basa en un temor a conectar afectivamente con el sufrimiento enloquecedor del paciente. Creo que a pesar de ser profesionales de la salud mental no estamos libres de caer en la locura. En el fondo podemos llegar a compartir muchas de las experiencias de sufrimiento de los pacientes más "locos". Entrar en contacto con esta realidad puede llegar a ser muy perturbador para el profesional, que se ve obligado a distanciarse afectivamente, escudándose habitualmente en el paternalismo y en la "relación profesional". Un ejemplo de ello es como los pacientes psicóticos controlados a nivel farmacológico son mejor soportados que los pacientes límites, con quienes la contratransferencia se hace incontrolable la mayoría de las veces. Distanciarse de un paciente con sintomatología negativa es fácil, no así con un paciente que nos hace sentir intensamente emociones como la angustia, la impotencia, la culpa, la agresividad o la inutilidad. Por otro lado, creo que el diagnóstico es otro de los mecanismos que muchas veces utilizamos para controlar y distanciarnos de los pacientes. A veces no somos conscientes de lo potente e invalidante que pueden llegar a ser comentarios como "este paciente es un límite", o "una histérica" o "un esquizofrénico crónico". Este tipo de comentarios suponen una "mirada" que determina significativamente nuestro trato con el paciente y nuestro nivel de conexión con su sufrimiento, antes de llegar a conocerle realmente.

Dentro de los espacios comunes con los pacientes, creo que es importante destacar era el taller de teatro. Para mí era un lugar privilegiado donde los roles defensivos de profesionales y pacientes quedaban completamente desarmados. Sin duda era un espacio de juego donde se creaba un ambiente de confianza y cooperación que permitía sacar lo mejor de cada uno. Nadie era tratado como paciente, los profesionales y los residentes participábamos como uno más. De hecho, la profesora prefería no estar en los grupos multifamiliares para evitar tener una visión de los participantes centrada en la patología o en el rol profesional. En este espacio cualquiera podía traer escritos propios para leerlos en público, o podía cantar y tocar canciones que se había preparado previamente. Pero sobre todo se permitía la improvisación a la hora de actuar y representar escenas de teatro. Gracias a estos ejercicios los múltiples self de los pacientes podían ser expresados sin sentirlos inadecuados o rechazables. Sin duda era un espacio donde el concepto de "virtualidad sana" adquiría toda su potencia. Los pacientes dejaban entrever aspectos sanos de ellos mismos, muchas veces impensables teniendo en cuenta el nivel de locura y sufrimiento que mostraban en otros contextos. Recuerdo un paciente muy ensimismado y desconectado del medio, con una intensa sintomatología psicótica que no remitía ni con el abordaje psicoterapéutico ni farmacológico. Fue increíble ver como este paciente se mostraba sorprendentemente espontáneo y afectivo cuando se le dejaba el espacio de teatro para cantar y tocar la guitarra.

\section{Los grupos multifamiliares.}

Estar en los grupos era toda una experiencia. Hasta ese momento estaba familiarizado con grupo con un encuadre muy definido, con un número de participantes reducido y con uno o 
dos coordinadores. Cuando entré por primera vez a un grupo multifamiliar me encontré a unas 40 personas sentadas en círculos concéntricos. Había hasta tres coordinadores, aunque no excluía la presencia de otros profesionales que también podía participar. Yo estaba en el grupo de residentes que solo observamos sin apenas intervenir, salvo en contadas ocasiones. El encuadre era flexible y abierto, los pacientes estaban obligados a asistir a las sesiones, pero habitualmente durante la sesión salían y entraban del grupo o se iban cambiando de lugar, siempre bajo la atenta mirada del equipo que se ocupaba de reintroducir o contener a los pacientes cuando se salían en momentos sensibles, o cuando se mostraban muy inquietos dentro de la sala.

Los pacientes designados y las familias se mezclaban hasta el punto de no ser distinguibles. Por ejemplo, hablaba una mujer muy perturbada que acaba siendo la madre de un paciente que permanecía callado en aparente calma y normalidad. Otra persona hablaba del daño que le había hecho su familia y acto seguido hablaba otra señora que resultaba ser su madre, la cual terminaba expresando su propio sufrimiento. En estas interacciones quedaba perfectamente representada la transmisión trans-generacional del trauma. Por otro lado, las identificaciones y transferencias entre los miembros del grupo eran muy potentes. Desde el lugar de padre, hermano o hijo todos se sentían reconocidos y validados de una manera u otra por las historias de sufrimiento de los otros. Pero las "presencias enloquecedoras" de todos los participantes quedaban activadas en esta dinámica, todos podían actuar desde el lugar de padre o de hijo, de objeto o de sujeto, de víctima o de agresor, de self sobreadaptado o de superyo sádico. El espacio que se creaba era de una riqueza y complejidad considerables.

Pero este espacio era ante todo un espacio de juego en el sentido que plantea Winnicott (1971). Las subjetividades se entrecruzaban y las experiencias de cada uno eran enriquecidas por el otro. Era un ambiente donde lo importante era la resonancia afectiva entre todos los participantes, más allá de la comprensión cognitiva y de las interpretaciones en busca de insight. La premisa principal para intervenir era el "hablar desde uno mismo", desde la propia experiencia. Poder compartir lo que se sentía en relación a la intervención del otro y como se llegaba a conectar con la propia historia. No se permitían "consejos" o interpretaciones, solo compartir aspectos vivenciales donde el otro pudiese reconocerse. El profesional seguía la misma premisa, cuando intervenía lo hacía desde su propia experiencia contra-transferencial cuando escuchaba al paciente. La implicación emocional era total, se introducían en el ambiente emocional del grupo y desde dentro facilitaban con su intervención y coordinación la elaboración de lo que estaba sucediendo. Por ejemplo, se facilitaban la intervención de algún paciente determinado que se creía que podía aportar algo significativo para otro en un momento dado. Creo que el principal instrumento de intervención del terapeuta era su actitud contra-transferencial tal y como la plantea Bollas (1987). Los ambientes emocionales eran la mayoría de las veces difícilmente pensados. Pero las sensaciones que uno iba sintiendo con el otro permitían guiar la intervención y crear un espacio potencial en el grupo. 
A pesar de todo habitualmente era difícil controlar y coordinar ambiente enloquecedor que muchas veces se creaban en los grupos. La intensidad de estas situaciones me hacía pensar en la exigencia emocional que soportan los profesionales día a día. En los grupos era donde había una exposición más directa a la parte más "loca" del paciente. No creo que un trabajo de este tipo se pueda llevar a cabo sin la idea de un equipo terapéutico bien coordinado. Las transferencias pasionales y psicóticas de los pacientes ponían constantemente en juego la capacidad de contención de los terapeutas. Ellos soportaban en el grupo el dolor y la agresividad que los pacientes depositan en ellos mismos cuando sus "presencias" se activan y se manifiestan con toda su crudeza. Por ello era necesario contar con el compañero para poder sobrellevar estas situaciones.

Creo que lo más importante del trabajo en equipo era llevar a cabo la función de tercero dentro del grupo, la cual permitía rescatar al compañero cuando este se veía envuelto en un enactment. Cuando se cierra toda posibilidad de comprensión mutua, es el tercero el que puede crear espacios en donde la experiencia de todos pueda ser validada y reconocida. Creo que los enactment entre un terapeuta y un paciente son inevitables, por ello es necesaria la presencia de un compañero que pueda sacar a los participantes de la actuación irreflexiva y llevar a un funcionamiento donde haya mayor reconocimiento mutuo. El compañero interviene para evitar que se invalide la experiencia del paciente en el enfrentamiento, evitando a la vez que este se sienta atacado. Todo ello sin que el terapeuta implicado sea desautorizado o descalificado cuando se explicita la confrontación y el conflicto.

Es una ilusión pensar que nosotros como profesionales podemos ser neutrales y no vernos afectados por nuestra historia y nuestras "presencias" cuando tratamos con pacientes. Todos los mundos de experiencia se interconectan en el grupo incluidos los residentes y los profesionales. Yo personalmente me he sentido en los grupos identificado con muchos pacientes, pero también he sentido un intenso rechazo por otros. He llegado a pensar que jamás podría tratar a ese tipo de pacientes individualmente, teniendo en cuenta las emociones que me suscitaban. Pero con la ayuda de un equipo creo que podría recibir el apoyo y supervisión necesarios para enfrentarme a estas situaciones sin miedo a caer en enactment irresolubles.

Cuando me planteo la importancia del equipo, lo pienso como un grupo humano más, y me llego a preguntar en los conflictos inevitables que pueden aparecer entre ellos, si son capaces de salirse de dichos conflictos, o si un miembro del equipo puede situarse como tercero para ayudar a otros compañeros en conflicto... También me planteo si los conflictos entre ellos se ven reflejados en el funcionamiento del grupo. Creo que cuando estos conflictos personales se filtran en el espacio grupal, puede provocar que un profesional no sea recatado adecuadamente por el compañero cuando se ve envuelto en un enactment con un paciente. Pero también puede provocar que delante de los pacientes se produzcan la devaluación o descalificación implícitas por parte de uno de los miembros del equipo de las actuaciones o decisiones que realiza otro compañero. Todo ello se podría resumir en la desconfianza entre 
los compañeros del equipo, lo que provoca que el propio profesional se vea obligado a encerrarse en una omnipotencia, pensando que solo él puede llevar el grupo o que podría llevarlo mejor que el otro. Esta soledad deja al profesional más expuesto a la "locura" del grupo, retroalimentando aún más su necesidad de omnipotencia para poder sobrellevar la intensidad emocional del día a día.

Todas estas posibles dificultades aparecen a nivel implícito y son difícilmente percibidas a simple vista, pero sin duda puede repercutir al grupo y en los pacientes. Por suerte fui testigo de cómo el poder auto-regulador y auto-organizador del grupo hace que estos problemas puedan ser superados. Pero siguiendo la filosofía de los grupos multifamiliares, me pregunto si una supervisión externa de todo equipo profesional de la salud mental ayudaría a solucionar más fácilmente los posibles conflictos con el fin de potenciar su funcionamiento. Sería positivo el poder contar con profesionales externos que pueda actuar como tercero y ayudar a disolver los enactment inevitables que aparecen entre los miembros de todo equipo de trabajo.

\section{Mi regreso a Madrid.}

A mi regreso a Madrid que me quedaba para finalizar mi residencia una última rotación de 4 meses en la interconsulta y la unidad de agudos del hospital. El primer día que llegué faltaban residentes de psiquiatría y uno de los psiquiatras. Ante la falta de personal se me pidió que fuese con el psiquiatra al quirófano. Iban a aplicar terapia electroconvulsiva (TEC) a una paciente de la planta y se necesitaba ayuda. Así que lo primero que hice nada más llegar a Madrid fue aplicar una descarga eléctrica al cerebro de una paciente que ni siquiera conocía. Teniendo en cuenta que venía de estar tres meses en un lugar donde se hablaba de la importancia de escuchar al paciente y de la "virtualidad sana", el contraste fue impactante y desconcertante. Pero no quiero ser ingenuo ni hacer un alegato en contra de este tipo de terapias. La paciente que había estado durante días casi en estado catatónico, de repente estaba hablando con compañeros y enfermeros horas después de la intervención. Sin duda este tipo de intervención puede ser justificable y de utilidad en casos extremos. En realidad, creo que lo que más me desconcertó fue cuando pregunté sobre la historia de la paciente. Nadie supo contestarme, solo me dijeron que tenía una depresión psicótica. Sentí que ese desconocimiento y esa etiqueta en cierta forma la podian deshumanizar. Creo que era muy importante explorar quien era esa persona realmente, más allá de verla de como una enferma mental grave a la que tratar médicamente.

Creo que tratamientos como la psicofarmacología y, en casos extremos, el TEC, no son tratamientos que deban ser rechazados. Creo que pueden ayudar si son aplicados de manera racional. A veces son muy necesarios cuando los síntomas agudos impiden el trabajo a nivel psicoterapéutico. Creo además que una medicación en un momento dado puede llegar a ser una "muleta" importante en el proceso terapéutico. El problema es como este tipo de 
tratamiento se convierte en un instrumento para situar al paciente en el rol de enfermo mental del que difícilmente puede desprenderse. En mi opinión el modelo médico basado en el diagnóstico y en la aplicar un tratamiento estandarizado, no es el adecuado para el tratamiento en salud mental. Esta forma de proceder impide muchas veces ver a la persona que tenemos delante, conectar con su sufrimiento y tratarle de una manera más humana. Muchos de los pacientes de los grupos multifamiliares estaban medicados, eran pacientes graves y era necesario ayudarles a contener su angustia también a nivel químico. Pero el objetivo era suministrar la dosis mínima necesaria para facilitar el trabajo grupal, basado en la relación y los vínculos emocionales con los otros. La medicación se planteaba como una ayuda no como la única solución al problema.

Creo que a mi vuelta a Madrid traje una idealización de lo que había vivido en mis tres meses en Argentina. Un lugar donde tratar al paciente de manera humana, realizando esfuerzos importantes por conectar con su sufrimiento y mostrando una disponibilidad emocional total. No se escatimaba en recursos y tiempo para dar un espacio de confianza y comprensión al paciente. La comparación con un hospital donde los recursos son limitados, facilitaba la idealización y aumentaba mi nivel de frustración por el trato que recibían los pacientes en este contexto. Creo que muchas veces este tipo de limitaciones implican una violencia para el paciente. Pero no hay que olvidar que el profesional también se ve obligado a acatar las condiciones que impone el sistema. A nivel institucional también se ejerce una violencia sobre el profesional que inevitablemente se trasmite al paciente. Se obliga a reducir el tiempo, los recursos, las sesiones y a la vez aumentar los resultados y las "altas clínicas". El trabajo psiquiátrico y psicoterapéutico de calidad no es valorado ni tenido en cuenta, da la sensación de que solo cuentan los números, no las personas.

Creo que al volver tenía la necesidad de hacer un duelo de lo vivido para evitar la frustración y el malestar que me provocaba la comparación. No creo que haya que caer en un pesimismo y en una queja permanente a pesar de la situación. Los recursos son los que son, tanto en el ámbito privado como en el público. Creo que lo importante es tener en cuenta como las limitaciones e implicaciones del contexto puede llegar a influir en nuestro trabajo con el paciente. Ser conscientes y mantener una reflexión constante en torno a ello nos permitirá ayudar de mejor manera y soportar con más calma y aceptación nuestras limitaciones. Sin duda este es uno de los mayores aprendizajes que he tenido en mi formación como psicoterapeuta y creo que marcará significativamente mi futuro profesional. 


\section{REFERENCIAS}

Aron, L. (1996). A meeting of minds. Mutualidad in psychoanalysis. New York: The Analytic Press

Bateson, G. (1971). Pasos hacia una ecología de la mente. Buenos Aires: Carlos Lohlé

Benjamín, J. (1996). Lazos de amor. Fenimismo y el problema de la dominación. Ed Paidos.

Benjamín, J. (1997). Sujetos iguales, objetos de amor. Ed. Paidos.

Bollas, C (1987) La sombra del objeto: psicoanálisis de lo sabido no pensado. Ed. Amorrortu. Buenos Aires.

Coderch, J. (2012). Psicoanálisis relacional de Frecuencia Semanal y Larga Duración (PRSLD). Bases teóricas y clínicas. Clínica e Investigación Relacional, 6 (3): 468-514. [ISSN 1988-2939] [Recuperado de www.ceir.org.es ].

García Badaracco, Jorge E. (1990). Comunidad terapéutica psicoanalítica de estructura multifamiliar. Editorial Tecnipublicaciones S.A.

García Badaracco, Jorge E. (2006a): "De sorpresa en sorpresa". Revista de Psicoterapia Analítica Grupal, Nro. 2, 2007.

García Badaracco, Jorge E. (2006b): "Virtualidad sana". Inédito.

García Badaracco, Jorge E. (2006e): "Psicoanálisis Multifamiliar, para curar la enfermedad mental desde la virtualidad sana". Revista de Psicoanálisis, LXII, 4, págs. 919-936.

García Badaracco, Jorge E. (2006f): "La nueva manera de pensar". Inédito.

Kohut, H. (1971). Analisis del self. Ed Amorrortu

Orange D. M., Atwood G., Stolorow R. (2012). Trabajando intersubjetivamente. Colección pensamiento relacional.

Selvini Palazoli, M; Cirillo, S; Selvini, M; Sorrentino, A. M. (1990): Los Juegos Psicóticos en la Familia. Ed. Paidos Terapia Familiar.

Segal, Hanna. (1989): Notas sobre la formación de símbolos. En la obra de Hanna Segal. Ed Paidós

Winnicott, D. (1956). La tendencia antisocial. En escritos de Pediatría y Psicoanálisis. Ed. Laia, España.

Winnicott, D. (1971). Realidad y juego. Barcelona: Gedisa.

Original recibido con fecha: 25/02/2016 Revisado: 28/02/2016 Aceptado: 28/02/2016 
Resumen:

En mi formación como psicólogo clínico he podido entrar en contacto con múltiples contextos clínicos y formativos. Al final de este periodo he podido participar en una comunidad terapéutica de psicoanálisis multifamiliar que me han permitido profundizar en la teoría de Jorge Luis García Badaracco y en la terapia grupal. Esta experiencia me ha permitido reflexionar sobre el significado que damos a la locura y a la enfermedad mental. Me ha hecho plantearme la manera de "mirar" a los pacientes con patología grave, dando una visión más humana de la experiencia psicótica y a los acting out. Con mi experiencia en la comunidad terapéutica he podido saber lo que es trabajar en un ambiente de mutualidad, donde la "vistualidad sana" del paciente constituye el aspecto más importante en el proceso terapéutico.

Palabras clave: Psicoanálisis Multifamiliar, grupos, psicosis, mutualidad, "virtualidad sana".

\section{Cita bibliográfica / Reference citation:}

Marín Arévalo, A. (2016). Explorando el Psicoanálisis Multifamiliar. Reflexiones en torno a la forma de entender el sufrimiento de los pacientes. Clínica e Investigación Relacional, 10 (1): 297-312. [ISSN 1988-2939] [Recuperado de www.ceir.info ] DOI: 10.21110/19882939.2016.100119 